\title{
Carnet de voyage
}

\author{
Ann Robinson
}

Travel Diary

A woman professor from Québec who is staying in Aix, records in a travel diary the process through which she comes to recognize herself as a lesbian. She sends letters to her lover and to her family; she keeps a journal; and she finds herself through reading: Marie-Jo Bonnet, Adrienne Rich and Alison Lurie. In such writing she finds confirmation of what had always existed in her on a latent level and what emerges now in all of its possibility.

\section{Samedi 15 avril 1989}

Bon, c'est fait. Me voilà partie, j'ai pris l'avion. Malheureusement, je suis dans la section non fumeuse. Le voyage sera long jusqu'à Aix, j'en ai bien peur. J'espère que tout ira bien là-bas. Avoir été choisie pour enseigner quatre cours sur Les femmes et les affaires dans la faculté de droit réputée la plus à droite de toutes les facultés françaises, ce n'est pas rien. Je devrai performer si je veux être invitée à nouveau. Enfin seule. Je peux commencer à décanter, à réfléchir à volonté et à satiété.

Nous nous sommes retrouvées, Première Amante et moi, plusieurs fois depuis décembre dernier. Quelles nuits! Je suis certaine qu'elle m'aime. $C^{\prime}$ est ma certitude à moi puisqu'elle ne $\mathrm{m}^{\prime}$ en a rien dit. Où vais-je dans tout cela? Franchirons-nous une autre étape à mon retour? Je devrais peutêtre reprendre ma lecture d'Un choix sans équivoque. Je suis ébranlée. On dirait que Marie-Jo Bonnet y décrit ce que j'ai ressenti lors des visites de Première Amante à l'Île d'Orléans : « [L]a passion n'est-elle pas une forme de folie coupée du monde des raisons? Seules au monde, les amoureuses ne connaissent qu'un seul langage : la défaillance des sens ». Est-il possible que personne ne s'en soit aperçu ? J'en doute. Mais combien de temps durera cette passion?

Dimanche 16 avril, $11 \mathrm{~h} 30$ heure de Paris

Je suis dans le train qui m'amène à Marseille. De là je devrai prendre un train de banlieue pour Aix. Cette journée finira-t-elle? Je m'endors, il n'est que 5 h30 pour moi. Le trajet entre Roissy et la gare de Lyon a été toute une 
aventure. Le train part. Je m'assoupis encore. Pas moyen de travailler. Tant pis pour mon cours de demain.

\section{Lundi 17 avril}

Je fais tout pour arriver à la faculté une demi-heure avant mon premier cours. J'y rencontre l'administratrice $\mathrm{M}^{\mathrm{me}} \mathrm{G}$. Première bonne nouvelle, je ne serai pas payée avant la fin de mon séjour. « Pas question » je rétorque, « je veux mon argent, j'en ai besoin ici et maintenant ». « On verra » ditelle. Mais elle m'explique que je dois d'abord ouvrir un compte bancaire puisque l'Éducation nationale versera mes honoraires dans ce compte. Pas de problème, ça, je connais.

Je ne suis pas nerveuse du tout, ça doit être ma témérité ou la fatigue du décalage. Ce premier cours est très général : influence des grands philosophes sur le droit positif, définition du principe d'égalité, application de cette définition aux règles de droit interne et international, etc. Retour à l'hôtel, je dors.

\section{Mardi 18 avril}

Course contre la montre. Je vais à la faculté puis je cherche une banque. J'en fais quatre; pas une ne veut ni de moi, ni de mon argent. Il doit bien y avoir quelque chose que je fais «tout croche». Mais quoi? Je m'ennuie du Québec. Ouf! À la fin de la journée, j'en trouve une. Il était temps! Je rentre à mon hôtel préparer le cours de vendredi. Il fait beau mais un peu froid. Je suis crevée, je mange à ma chambre, pain, fromage et bière. C'est tout ce que je peux m'offrir.

\section{Vendredi 21 avril}

Insignifiante que je suis. Pourquoi ne suis-je pas tranquillement en train de me reposer à la maison après un gros trimestre? J'ai la " chienne » de donner ce deuxième cours. Pourquoi je me mets sans arrêt dans des situations impossibles? Est-ce toujours aussi compliqué de vivre et de faire carrière pour une femme?

Finalement je me paie un steak-frites dans un bar, quel luxe! Puis, à la chambre, première bouteille de vin. Le repos de la guerrière! C'est pas trop tôt.

\section{Samedi 22 avril}

Journée récupération. Je fais la grasse matinée jusqu'à 8 h30. Je termine toutes sortes de petits travaux laissés en plan avant mon départ de 
Québec. Je prends quand même le temps de repenser à mon cours d'hier après-midi. Au premier cours, j'avais 22 étudiantes et étudiants présents et au deuxième cours, je n'en n'ai que 6 . Encore que ce deuxième cours était plutôt politique et pas trop féministe puisqu'il portait sur la notion de discrimination, sur les chartes canadienne et québécoise, sur les aspects historiques du partage des compétences entre le fédéral et les provinces, et sur la situation des femmes en général dans ce contexte politique. L'intolérance est à son meilleur en France. Tiens, tiens, au prochain cours, je leur parlerai de L'Intolérance de Lise Noël.

Puis je vais me promener dans un magnifique parc au centre d'Aix. Il fait beau. Je suis presque seule. J'ai envie d'écrire à Première Amante. J'aimerais qu'elle soit là. J'ai relu sa carte hier. Elle dit qu'elle tient à moi... Quel euphémisme!

J'aime Première Amante, peu importe qu'elle soit homme ou femme. Je l'aime, c'est un être humain « aimable ». C'est ainsi que j'y songe depuis quelques mois, mais ma réflexion commence à aller plus loin. Je l'aime peut-être ... parce qu'elle est femme.

Plusieurs événements m'amènent à me l'avouer.

La dernière année de vie commune avec mon ex-conjoint, faire l'amour devient toute une expédition, beaucoup de vin, longue préparation, question $\mathrm{d}$ 'hygiène mentale et physique. Pourtant je n'ai pas l'idée de prendre un amant. MA LIBIDO EST EN VEILLEUSE.

C'est l'époque où je donne le cours Femmes et droit et où je suis entourée de femmes. C'est aussi l'époque où je commence à lire sur l'homosexualité d'abord, puis sur la sexualité des femmes et enfin sur le lesbianisme. Je suis malgré tout bien dans ma peau. MA SEXUALITÉ EST EN VEILLEUSE.

Une nuit d'octobre 1987, je prends conscience que je veux à tout prix me retrouver seule, mon deuxième conjoint doit me quitter, sinon c'est le suicide tant sa présence dans ma vie est devenue problématique. Une seule certitude. PLUS JAMAIS D'HOMME DANS MON QUOTIDIEN.

Mais je ne cherche toujours pas d'amant, je suis toujours entourée de femmes, tant hétérosexuelles que lesbiennes. Surtout de celle qui deviendra bientôt Première Amante. Commence alors l'époque de nos téléphones sans fin, nos cafés prolongés. Je lui disais tout: mes angoisses, mes peines et ma libido toujours en veilleuse. Ma sexualité dort toujours. JE NE ME FAIS MÊME PLUS L'AMOUR.

Quelques mois passent, puis c'est l'explosion. Un soir de grandes confidences, je « connais » enfin Première Amante! Quel bonheur! Quelle 
volupté! Quels plaisirs! Mais aussi quelles douleurs! Quelles souffrances! Amoures clandestines et amoures défendues, que je vous hais alors! Mais le bonheur et le plaisir sont sans doute plus forts que la souffrance. Quand je regarde ses yeux, son visage, je suis transportée. Je l'aime comme je n'ai jamais aimé, même mon premier conjoint. Faire l'amour avec une femme, partager son intimité, c'est comme se détendre au bord de la mer après une traversée du Sahara à dos de chameau. J'AIME UNE FEMME.

Mais voilà que depuis mon arrivée à Aix, je me sens toute bouleversée, je crois que je ne suis plus hétérosexuelle, pas même bisexuelle. JE SUIS LESBIENNE. Voilà, le mot est lâché! J'en ai des frissons à la fois ... d'horreur et de volupté.

\section{Mardi 25 avril}

Je me lève tôt. Je cours à la faculté déposer mon premier relevé bancaire récupéré hier. $\mathrm{M}^{\mathrm{me}} \mathrm{G}$. est trop heureuse de m'annoncer qu'ainsi j'aurai droit à une avance de $70 \%$ de mes honoraires ... dans 21 jours. Eh bien! Dans 21 jours je serai retournée chez moi à Québec mais je manquerai d'argent dans environ 10 jours. Elle s'agite, fait quelques appels, fume une ou deux cigarettes, puis elle me dit : "Alors, devancez votre départ. " Je lui réponds : "C'est impossible, j'ai des cours les 9 et 11 mai. " Elle enchaîne : «Annulons les cours. » Rien de moins!

Je ne veux pas devancer mon départ à cause de la trésorerie française, c'est trop bête. J'ai le dernier mot : « De toute façon, j'ai un billet fermé. Si je devance mon départ, des frais me seront réclamés.» Je sens qu'elle me trouve bien miteuse. Je suis imperturbable, des problèmes de sous, toute ma vie j'en ai eu. La faculté me doit de l'argent, qu'elle s'arrange pour m'en trouver. Elle finit par me dire : «Écoutez, on fait le point dans une semaine et si vous n'avez rien reçu quelqu'un ou la faculté vous fera un chèque personnel. »

\section{Jeudi 27 avril}

Une boîte postale pleine de livres et de documents sous le bras, je quitte mon hôtel. C'est lourd mais je suis contente, une lourdeur de moins pour mon voyage de retour. J'arrive à la poste, le commis fait celui qui ne comprend pas. « Pas plus d'un kg. Non madame, je ne peux pas. » Alors, je lui dis qu'on m'a parlé d'un sac postal de librairie il y a quelques jours. Il consulte à droite et à gauche, et finit par me remettre un sac de jute. Je jubile, j'ai réussi. Je prends la boîte, je la scelle, je la glisse dans le sac, j'écris 
mon adresse sur une feuille bleue, tel que prescrit. Je retourne porter le tout à la caisse de mon commis. Il me regarde alors comme si j'étais une demeurée. Furieux, il me dit : « Mais, madame, il faut ficeler le sac. Et la feuille bleue, cela ne va pas du tout, ce n'est pas assez rigide. Déjà que vous avez un tarif réduit pour les livres, vous pourriez faire un effort. »

Personne ne m'avait dit qu'il fallait ficeler le sac et que " n'importe quoi de bleu », excluait une feuille bleue. Je dois être habitée par la Grande Déesse, je reste imperturbable. "Bon d'accord, vous me gardez mon paquet, moi je vais chercher ficelle et carton bleu. " J'avais repéré une quincaillerie. J'y cours, je trouve ficelle et ciseaux-pour couper la ficelle bien sûr-. Il faut ensuite que je trouve une imprimerie pour acheter le carton bleu. Je l'ai.

De retour à la poste, je reprends mon paquet, le ficèle solidement et y accroche mon carton bleu. Je rapporte le tout au commis avec un sourire de victoire et je lui dis que j'aurai 2 ou 3 autres boîtes d'ici deux semaines, pensant le faire suer. Alors il me répond cyniquement: «Dans ce cas madame, il faudra faire peser vos boîtes à la poste, parce que si vous $\mathrm{n}^{\prime}$ avez que $5 \mathrm{~kg}$. et moins, on peut prendre les boîtes. La vôtre ce matin faisait quelques grammes de trop, voilà pourquoi vous avez dû utiliser le sac de librairie. »

L'écoeurant! Il a eu le dernier mot. Pour quelques grammes de trop! Je l'aurais tué!

\section{Vendredi 28 avril}

J'erre dans la ville comme une âme en peine. Il fait beau mais la solitude me pèse de plus en plus. Qu'est-ce que je donnerais pour avoir une oreille attentive à qui me confier, raconter mes amoures impossibles? Ah, que voilà une belle idée!

\section{Lettre à ma sœur,}

Il y a déjà presque deux semaines que je suis à Aix. Je me repose bien, je lis, je travaille et surtout je réfléchis. Il y avait bien longtemps que je n'avais pas eu le temps de faire le vide en moi, revoir mon passé et projeter mon avenir.

Tu seras sans doute surprise de recevoir cette lettre. J'ai choisi de te l'écrire à toi et pas aux autres parce que j'ai pensé que tu serais sans doute la mieux préparée pour recevoir ce que j'ai à vous dire, à vous membres de ma grande famille. Je $n^{\prime}$ irai pas par quatre chemins. Depuis un certain temps, je cherche ce qui m'arrive, pourquoi j'ai tant besoin de solitude, pourquoi je suis toujours tellement mieux seule, et pourquoi même les enfants me pèsent de plus en plus. 
J'ai tenté de renouer avec mon premier conjoint. Nous nous sommes vus quelques fois et nous avons fait l'amour. Mais ces moments, au lieu de m'apaiser, $m^{\prime}$ ont rendue encore plus taciturne. Je vais cesser de le voir.

En toute franchise, je ne peux renouer ni avec lui, ni d'ailleurs avec aucun autre homme, parce que je suis déjà amoureuse ... d'une femme.

Je te vois et je t'entends déjà argumenter. Me dire que je suis fatiguée. Tu me diras que je suis frustrée par les hommes que j'ai rencontrés. À cela je répondrai que j'ai eu au moins 30 amants, dont deux maris en moins de 22 ans. Selon les lois de la probabilité, il serait difficile de maintenir cet argument.

Tu me diras que c'est une mauvaise passe, que c'est la ménopause. À cela je répondrai que je suis contente d'être enfin arrivée là parce que je commence à peine à vivre. Je me reconnais, je me comprends mieux et je peux expliquer certains comportements de jeunesse.

Je n'ai jamais eu envie de fréquenter les garçons. Tu te souviens, toi et les autres avez bien assez ri de moi à ce propos. J'ai choisi mon premier mari dès les premières semaines de cours à l'université, me protégeant ainsi des autres garçons de la classe. Bien sûr je l'aimais, je ne suis pas masochiste, mais mon désir obsessionnel compulsif d'avoir des bébés pour vous prouver que j'étais une vraie fermme a vite pris le dessus sur tout le reste.

Je ne suis ni une invertie, ni une déviante, ni une perverse. Je suis une femme qui a enfin trouvé ce qui la trouble depuis si longtemps. Je me rappelle mon envie de "rentrer chez les bonnes sceurs » après ma $12^{e}$ année. Je le voulais vraiment parce que je n'avais que faire de ce monde que je ne comprenais pas et qui ne me comprenait pas. Par la suite, j'ai fait tout ce que j'ai pu pour étudier le plus longtemps possible, pour être dans mes livres hors du monde.

Tu peux en parler aux autres, cela m'est égal. D'ailleurs il faudra bien que vous vous fassiez à l'idée, je suis lesbienne, c'est ma vie désormais. Ton dernier argument, je le connais : les enfants. Que diront-ils? Comment vivront-ils cette dernière folie de leur mère? Tout d'abord, les enfants en général et les miens en particulier sont plus coriaces qu'on ne le pense. I'ai toujours eu avec eux une relation de franchise, je leur ai toujours tout dit. Ce qu'ils veulent les enfants, c'est que je sois heureuse. Et s'ils et elles constatent que je le suis, cela leur suffira avec le temps, $j^{\prime}$ en suis certaine. Après tout ce sont des adolescents et adolescentes.

Ne t'en fais pas, je suis seule à Aix, je me sens bien, je peux réfléchir à volonté. Sois heureuse.

\section{Dimanche 30 avril}

Je suis attablée dans un restaurant à l'aéroport de Bruxelles. Il est déjà 20h30. J'ai bouffé une énorme salade de poulet, c'est mon premier repas de 
la journée. Et j'ai devant moi un verre de bière tellement grand, qu'il ferait pâlir d'envie mon fils Blaise. Vrai comme je suis là, il doit faire au moins $30 \mathrm{~cm}$. de haut et peser $1 \mathrm{~kg}$.

Je pars tantôt pour l'Afrique afin de participer à un colloque en pédagogie universitaire sur le thème de la pédagogie aux grands groupes. Quelle histoire! Aix/Marseille/Paris/Bruxelles, en train. Partie ce matin d'Aix à $7 \mathrm{~h}, \mathrm{j}$ 'avais vraiment $\mathrm{l}^{\prime}$ impression d'avoir respecté toutes les règles puisque personne ne m'avait encore rien dit jusque dans le train Paris-Bruxelles. Horreur, le contrôleur s'est rendu compte que je n'avais pas composté mon billet. Je fais l'imbécile et lui dis de mettre lui-même la date sur le billet. J'ai dû avoir un air convaincant car il a été plus poli par la suite et m'a expliqué pourquoi il fallait que j'inscrive la date moi-même. Je n'ai pas vraiment compris mais je lui ai renvoyé un large sourire. Tout est rentré dans l'ordre.

Je suis fatiguée. Mais je me sens mieux en Belgique qu'en France. C'est plus relax, plus simple.

Tout ce que j'espère maintenant, c'est que les collègues du Québec arrivent à temps pour que nous puissions continuer ensemble le voyage vers Bujumbura cette nuit. Plus de 30 heures de voyage!

\section{Lundi $1^{\mathrm{er}}$ mai}

Le colloque ne commence que demain. J'en profite pour me reposer et lire. Je viens tout juste de finir Adrienne Rich, Naître d'une femme. Je suis bouleversée. Son analyse de la maternité, de la filiation, du patriarcat m'a laissé le goût de continuer à cheminer, à réfléchir, à aller plus loin. Mais mon bouleversement vient surtout du fait que certaines parties de son livre décrivent si bien mes états d'âme.

Tout l'hiver j'ai pensé à Première Amante et j'ai souffert de nos séparations plus ou moins prolongées. Et maintenant si loin, si longtemps sans la voir, la toucher, la sentir, l'embrasser partout. J'aurais tellement aimé découvrir avec elle ce pays d'Afrique. «[Q]uand chaque séparation de la vie et de chaque jour nous vole de nos moments », écrit Rich. Voilà le reflet de mon âme, le miroir de mon amour pour Première Amante.

\section{Mardi 2 mai}

Mardi déjà. Le colloque est commencé. Il fait chaud et humide : environ $32^{\circ} \mathrm{C}$ avec $80 \%$ d'humidité. On est bien logé, dans un hôtel Méridien à Bujumbura, climatisé avec tout le confort nord-américain. 


\section{Jeudi 4 mai}

Aujourd'hui, c'est l'Ascension. Comme le Burundi est un pays où le catholicisme est dominant, c'est jour férié. Nous avons travaillé un peu ce matin. Cet après-midi, excursion à Resha, au bord d'un très grand lac. $\mathrm{Ce}$ lac me rappelle le fleuve. J'ai la nostalgie de la maison. Je suis bien installée et je rêve puisque j'ai oublié de m'apporter un livre. Il fait très chaud, mais il y a une petite brise. Déjà jeudi. Le temps passe plus vite ici qu'à Aix.

La semaine dernière j'avais un petit cafard, le Québec me manquait, la maison, les enfants et Première Amante. Je suis allée fureter dans les librairies. Ne sachant trop ce que j'avais envie de lire, j'ai choisi un roman écrit par une Américaine que je ne connaissais pas, Alison Lurie, La vérité sur Lorin Jones . Bien ou mal m'en prit? Je n'en sais rien. Je l'ai terminé hier soir et j'en suis toute remuée.

La description de la vie de Polly la narratrice, ressemble en plusieurs points à la mienne. $C^{\prime}$ est une femme de quarante ans qui, divorcée depuis 18 mois, rejette l'hétérosexualité comme forme d'expression de sa sexualité parce qu'elle considère que les hommes sont tous sexistes et machos. De toute façon, depuis son divorce, sa libido est en veilleuse et elle ne se plaint pas. Et en fait, elle est entourée de femmes toutes plus ou moins lesbiennes. Et ce qui devait arriver, arriva. Elle couche avec sa meilleure amie, sa confidente de toujours. Mais elle n'est pas satisfaite sexuellement avec Jeanne.

Et toi, Première Amante, pourquoi m'as-tu entraînée dans cette aventure aussi folle? Comme Jeanne l'explique à Polly, pour me faire du bien? Ou, comme tu me l'as souvent répété, pour servir d'élément catalyseur dans ma vie?

Mais je me réjouis d'avoir été satisfaite toutes les fois où j'ai fait l'amour avec Première Amante. Mon exultation dépasse toujours largement la plupart de mes expériences hétérosexuelles. Voilà ce qui me bouleverse le plus. Bien sûr j'aime Première Amante, mais je crois qu'il y a plus : lorsque je fais l'amour avec elle, je ressens une sensation de plénitude que je n'ai jamais ressentie avec aucun homme. Et je l'ai vécue dès la première fois, à mon grand étonnement d'ailleurs.

Burundi, paradis terrestre. Comparable à la plus belle nuit de juillet à l'île d'Orléans, une nuit chaude et sans nuage, des milliers d'étoiles. C'est ainsi toutes les nuits dans la vallée de Bujumbura. De mon balcon, tous les soirs je contemple le ciel et je rêve. Dis-moi, Première Amante, pourquoi ne partirions-nous pas toutes les deux au bout du monde vivre une longue, 
longue parenthèse? Je rêve, je rêve, je rêve ... Le côté exotique et irréel de ce court voyage, m'a rendue complètement euphorique.

\section{Lundi 8 mai}

De retour à Aix après plus de 24 heures de voyage. Complètement crevée. Il me reste deux cours à donner avant de revenir au Québec. Plus que 8 jours. Je commence à sentir des chatouillements tout partout. MA LIBIDO N'EST PLUS EN VEILLEUSE.

Première Amante me manque tellement. J'ai mal. Je n'arrive même plus à exprimer ce que je ressens pour elle. Je vois une tortue qui, sentant le danger approcher, se recroqueville dans sa carapace pensant qu'elle ne risque plus rien. Mais même les tortues peuvent être apprivoisées, par qui sait s'y prendre. Le saurai-je?

Je suis bien consciente d'être dans un autre virage en tête d'épingle de ma vie. LE GRAND VIRAGE. J'accepte d'être une femme avec un corps qui l'habite pleinement, et un désir de partage que je ne réussirai sans doute jamais à combler entièrement avec Première Amante. Tant pis, pour le moment j'ai juste envie d'être quelque part à Québec avec elle, je voudrais qu'elle me pousse dans mes derniers retranchements comme elle seule sait le faire, qu'elle soit dans ma vie quoiqu'il arrive. Elle est jeune, elle prend la vie à pleines mains, j'aime cela.

\section{Samedi 13 mai}

Plus que trois jours avant mon départ. J'avoue qu'Aix me pèse un peu. $C^{\prime}$ est une petite ville de province qui ne vaut peut-être pas qu'on s'y arrête un mois. Enfin, il faut dire que l'adrénaline est tombée depuis que tous mes cours sont donnés. Je ne peux pas dire que j'ai redoré mon blason à l'occasion des deux derniers : l'un portait sur l'accès à l'égalité et l'autre sur le harcèlement sexuel. J'avais à peu près une vingtaine d'étudiants et étudiantes pour cette dernière prestation. Vous dire tout ce que j'ai entendu comme sarcasmes sur le harcèlement sexuel en milieu de travail! Mais j'étais déjà ailleurs. Tout ce qui compte $c^{\prime} e s t$ que je rentre chez moi dans quelques jours. J'y retrouverai mes enfants, mon travail, ma maison, mes amies. Et ma ... blonde? 